\title{
Mechanical Comparison of High-Strength Tape Suture Versus High-Strength Round Suture
}

\author{
William P. Ensminger, M.D., Terence McIff, Ph.D., Bryan Vopat, M.D., Scott Mullen, M.D., \\ and J. Paul Schroeppel, M.D.
}

\begin{abstract}
Purpose: To compare knot and loop characteristics of commonly used high-strength tapes and high-strength round sutures. Methods: Twenty tied 30-mm loops were prepared for using suture-knot combinations of 4 common arthroscopic knots or a hand-tied surgeon's knot and 7 sutures. Two tapes (BroadBand, SutureTape) and three no. 2 sutures (MaxBraid, FiberWire, DynaCord) were compared. Two additional larger tape sutures (FiberTape, PermaTape) not commonly tied arthroscopically were included in the hand-tied group. Each suture-knot combination was evaluated for loop integrity (single load to a specified elongation), knot security (plastic loop deformation with cyclic testing), loop security, and knot volume. Data were compared using analysis of variance followed by Tukey-Kramer post hoc tests. Results: BroadBand and SutureTape demonstrated superior loop integrity compared to most no. 2 suture-knot combinations. FiberTape $(430.48 \pm 89.00 \mathrm{~N})$ and PermaTape $(545.16 \pm 95.09 \mathrm{~N})$ required significantly greater force to undergo $3 \mathrm{~mm}$ of displacement than all other hand-tied sutures $(P<.001)$. All suture-knot configurations demonstrated significant plastic deformation (cyclic elongation) over 1000 cycles $(P<0.001)$. DynaCord displayed the best loop security except when paired with the Duncan loop. Overall, BroadBand and SutureTape had lower knot volumes compared to all other sutures. Conclusion: This study exhibits the ability of narrow tape sutures to provide reliable tissue apposition and fixation in multiple suture-knot configurations, while decreasing the potential adverse effects accompanying knot prominence. Generally, BroadBand and SutureTape demonstrated an ideal balance of mechanical characteristics to achieve the optimal suture-knot construct among those tested. Dynacord displayed superior loop security and performed best among round sutures. PermaTape and FiberTape showed greater loop integrity, albeit to the detriment of loop security and knot volume. Clinical Relevance: When securing a rotator cuff repair construct with a knot, one should have a basic understanding of the mechanical properties of the suture and knot being used to provide the best possible repair. This study will provide information about the mechanical characteristics of commonly used high-strength tape suture knots and loops to help determine what knot type may provide the best overall construct.
\end{abstract}

$\mathbf{I}_{\mathrm{j} w}^{\mathrm{n}}$ $\mathrm{n}$ arthroscopic and open treatment of soft tissue injuries, a strong, stable repair is necessary to optimize healing potential and postoperative outcomes. With

From the Department of Orthopedic Surgery, University of Kansas Medical Center, Kansas City, Kansas, United States.

The authors report the following potential conflicts of interest or sources of funding: Funding provided by the Marc A. and Elinor J. Asher Orthopedic Research Endowment. J.P.S. reports personal fees from Vericel Corporation. S.M. reports personal fees from Stryker Sport Medicine. B.V. reports personal fees from DePuy, a Johnson $\theta$ Johnson company, and other from Stryker. Full ICMJE author disclosure forms are available for this article online, as supplementary material.

Received January 6, 2021; accepted July 16, 2021.

Address correspondence to William P. Ensminger, M.D., University of Kansas Medical Center, Department of Orthopedic Surgery, 3901 Rainbow Blvd., Mailstop 3017, Kansas City, KS 66160,U.S.A.E-mail:wpensminger@ gmail.com

(C) 2022 THE AUTHORS. Published by Elsevier Inc. on behalf of the Arthroscopy Association of North America. This is an open access article under the CC BY-NC-ND license (http://creativecommons.org/licenses/by-nc-nd/4.0/).

2666-061X/2123

https://doi.org/10.1016/j.asmr.2021.07.014 advancements in implant technology and surgical techniques, results of operative treatment have generally improved over time; however, failure of surgical repairs continue to occur.

In rotator cuff repairs, for example, retear rates of large tears remain relatively common, ranging from $10 \%$ up to $41 \%$ at 6 months, and up to $90 \%$ at 1 year for massive cuff repairs. ${ }^{1,2}$ The introduction of suture anchors and double-row techniques attempted to improve these results. Additionally, the development of high-strength tape sutures led to improved biomechanical characteristics of the repair construct. The tape design provides increased tendon-footprint contact pressures and greater suture-tissue pullout strength compared to highstrength round suture. ${ }^{1-5}$

Ultimately, the tissue-suture repair construct must be placed in a manner that will provide immediate durable fixation of the soft tissue to bone and remain stable until adequate healing has occurred.,.$^{3,6-9}$ When 
securing the repair construct with a knot, one should have a basic understanding of the mechanical properties of the suture and knot being used to provide the best possible repair., ${ }^{3,12}$ Many studies have compared multiple knots, including arthroscopic and open, with various round no. 2 sutures; however, little exists regarding the characteristics of knot and loop performance in high-strength tape suture.

It has been implicated that suture or material-related failure results from a single extreme load, while biologic or tissue failure occurs via cyclic loading. 9 ,13-16 Numerous studies have affirmed the importance of knot and loop security to create an effective knotted suture loop construct. ${ }^{6,7,11,13-24}$ Loop security refers to the ability to maintain a fixed suture loop while tying the knot. ${ }^{6,16,17}$ Knot security has been defined as the knot's ability to resist slippage (or breakage) and is dependent on factors including friction, internal interference, and amount of slack between throws. ${ }^{11,16,19,21,22,24,25}$

Single-load strength testing is a commonly used method to quantify knot security. ${ }^{13,16,18,19,21,22,24,26,27}$ However, this fails to account for the material properties of the suture, which will elongate based on its innate elasticity. ${ }^{20,23,24}$ Therefore, single load strength testing characterizes the summation of knot slippage, or knot security, and the elastic deformation of the suture. The authors of this study refer to this as loop integrity.

Knot security was better characterized by the effects of cyclic testing. ${ }^{20,28}$ The amount of plastic loop deformation after 1000 cycles, referred to as cyclic elongation, more accurately isolated knot slippage, despite material (suture) failure typically occurring as a result of a single, sustained force. ${ }^{9,13,16}$

Knot volume can also influence surgical outcomes. Symptomatic prominence of suture material, typically involving the knot, has been described. ${ }^{7,8,10,11,14,15,29-31}$ Authors have noted knot prominence causing irritation after rotator cuff procedures because of minimal clearance beneath the acromion. ${ }^{31}$ Injury to the articular surface can ensue if a knot is positioned in close proximity. Placement of large prominent knots adjacent to a joint surface is a described cause of chondral injury after shoulder labral repairs and knee meniscal repairs. ${ }^{14}$ Foreign body reaction is also of concern and can be exacerbated with increasing amounts of suture material present. $7,8,11,14$

The purpose of this study was to compare knot and loop characteristics of commonly used high-strength tapes and high-strength round sutures. The authors hypothesized that the narrow tape sutures would demonstrate overall superior mechanical characteristics across all knot types to provide the best overall sutureknot construct.

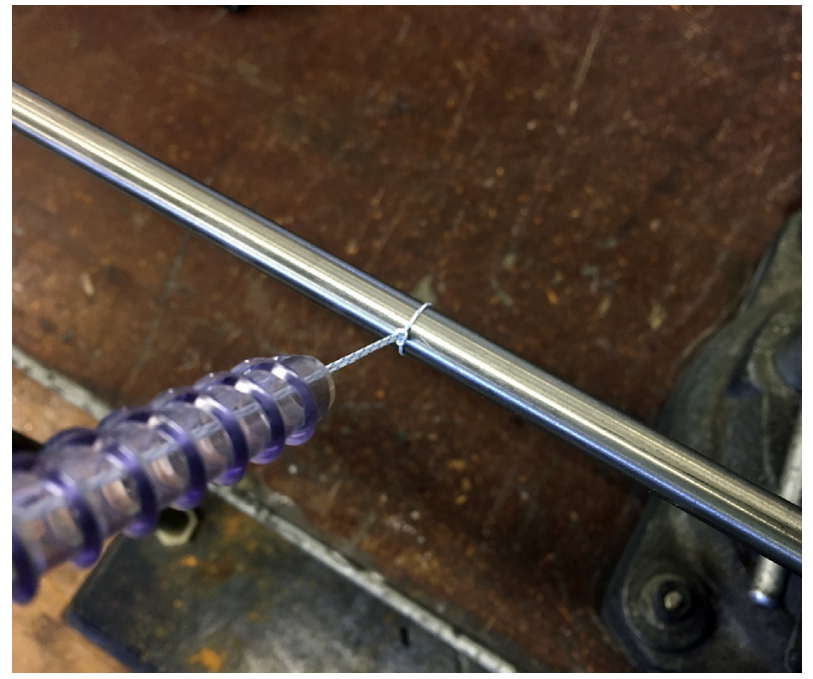

Fig 1. Knot-tying cannula setup. Knots were tied through a standard arthroscopic cannula using a knot pusher, onto the 30-mm dowel.

\section{Methods}

Four high-strength tapes and three high-strength round sutures were tested. The tapes were BroadBand (Zimmer-Biomet, Warsaw, IN), SutureTape (Arthrex, Naples, F:), FiberTape (Arthrex), and PermaTape (DePuy-Mitek, Raynham, MA). The widths measured $1.3 \mathrm{~mm}$ for BroadBand and SutureTape, whereas FiberTape and PermaTape were $2.0 \mathrm{~mm}$ and $2.5 \mathrm{~mm}$ wide, respectively. The no. 2 sutures consisted of MaxBraid (Zimmer-Biomet), FiberWire (Arthrex), and DynaCord (DePuy-Mitek).

Four commonly used arthroscopic knots were tested: the arthroscopic surgeon's knot, Samsung Medical Center (SMC) knot, Tennessee slider, and Duncan loop. Each knot was secured with 3 reversing half-hitches on alternating posts. ${ }^{32}$ The surgeon's knot was also tested using the standard open 2-hand tie technique and secured with 3 alternating square throws for a total of 5 throws. To simulate intraoperative conditions, all knots were tied while wearing surgical gloves with saline solution-soaked sutures. The arthroscopic knots were tied through an arthroscopic cannula using a knot pusher (Fig 1). ${ }^{13,33}$ Tape designs measuring $2 \mathrm{~mm}$ or greater in width are not commonly tied arthroscopically and were tested only with the hand-tied surgeon's knot. All arthroscopic knots were tied by a single sports medicine fellowship-trained orthopaedic surgeon. All hand-tied knots were tied by a single senior-level orthopaedic resident. Sutures were presoaked for several minutes before being tied over a stainless-steel dowel, creating a $30-\mathrm{mm}$ suture loop. The $30-\mathrm{mm}$ loop was chosen in part to be consistent with other studies and to reduce the elastic effects of the suture material by keeping the loop relatively small. ${ }^{13,21,22,24,33,34}$ 


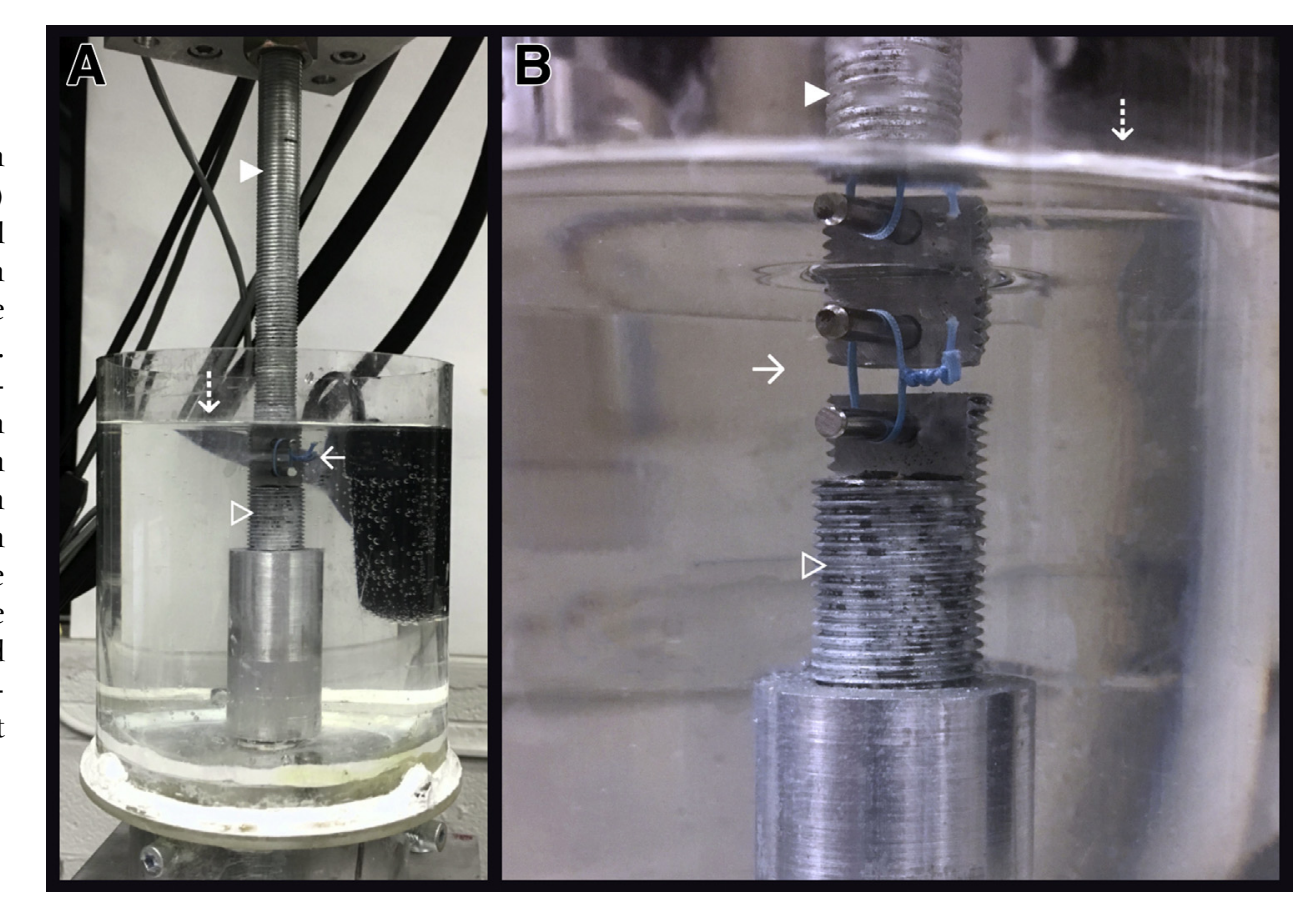

Fig 2. Material testing system apparatus. (A) Overview and (B) Enlarged image of the mechanical testing equipment. The upper arm (solid arrowhead) is affixed to the testing machine actuator above. The lower arm (hollow arrowhead) is attached to custom fixture within the saline bath (dashed arrow). The custom fixture and saline bath are atop an $\mathrm{X}-\mathrm{Y}$ table (not pictured) to ensure inline pull during testing. The solid arrow denotes a knotted suture loop held by two stainlesssteel posts, as it would appear just before testing.

Mechanical testing was performed using a servohydraulic materials testing machine (MTS Systems, Minneapolis, MN). For testing, each knotted suture loop was carefully removed from the dowel and placed over two 3.15-mm-diameter stainless-steel posts for single load or cyclic testing. The upper arm was affixed to the testing machine actuator and the lower arm was connected to a custom-made fixture submersed within a $37^{\circ} \mathrm{C}$ isotonic saline bath (Fig 2). ${ }^{20,24}$ The bath and fixture were supported by an X-Y table that could slide to ensure alignment of the direction of suture pull with the tensile force applied. ${ }^{20}$ The suture loops were pretensioned to $10 \mathrm{~N}$ once placed over the posts. The loop length at the pretensioned load then served as the starting position for the single load and cyclic tests. ${ }^{12,13,25,26}$ In total, 400 arthroscopic knots and 140 hand-tied knots were tied and used for testing with single load-to-failure or cyclic loading.

\section{Single Load Testing}

The force required to elongate the knotted loop $3 \mathrm{~mm}$ during single-load testing was the primary outcome measure of the study. Each suture loop was loaded to failure at a rate of $60 \mathrm{~mm} / \mathrm{min}$ or 2 times the length of the loop being tested, in accordance with the U.S. Pharmacopeia standards for suture tensile strength testing. ${ }^{9}$ Failure load was defined as the maximum force reached during upward crosshead displacement from 0 to $3 \mathrm{~mm}$ (loop elongation) or at knot breakage before reaching $3 \mathrm{~mm} .{ }^{12,13,25,34}$ Maximum tensile force at $3 \mathrm{~mm}$ of elongation was recorded. Results with failure defined at $2 \mathrm{~mm}$ were also evaluated. ${ }^{13,34}$ Each suture-knot combination was tested using 10 specimens in single load testing to reduce variability and to remain consistent with previous studies. ${ }^{7,8,14,26,27,33}$

\section{Cyclic Testing}

Each suture loop was loaded with a sinusoidal wave form from 10 to $60 \mathrm{~N}$ at $1.0 \mathrm{~Hz}$ for 1000 cycles. Cyclic elongation was recorded at the end of cyclic testing and defined as the amount of plastic loop deformation, following initial pre-tensioning, that occurred after 1000 cycles of loading. The knotted suture loops were then loaded to failure using the single load testing protocol. ${ }^{12,13,25,26}$ Knot breakage or elongation of $3 \mathrm{~mm}$ at any point during cyclic testing was considered a failure. Each suture-knot combination was tested using 10 specimens in cyclic load testing.

\section{Loop Security}

Loop circumference was measured after pretensioning to evaluate initial loop security using the formula previously described by Lo et al. ${ }^{21}$ in 2004. Because each loop and knot was tied around a dowel having a 30-mm circumference, perfect loop security was assumed to yield a pretensioned loop length of 30 $\mathrm{mm}$. Each suture-knot combination was evaluated using 20 specimens before cyclic or single load testing.

\section{Knot Volume}

To assess knot size, the knot volume was calculated by measuring the height and depth of each knot before testing, on the basis of scaled photography and using Adobe Photoshop software (Adobe Systems, San Jose, CA). The formula used to measure knot volume was 


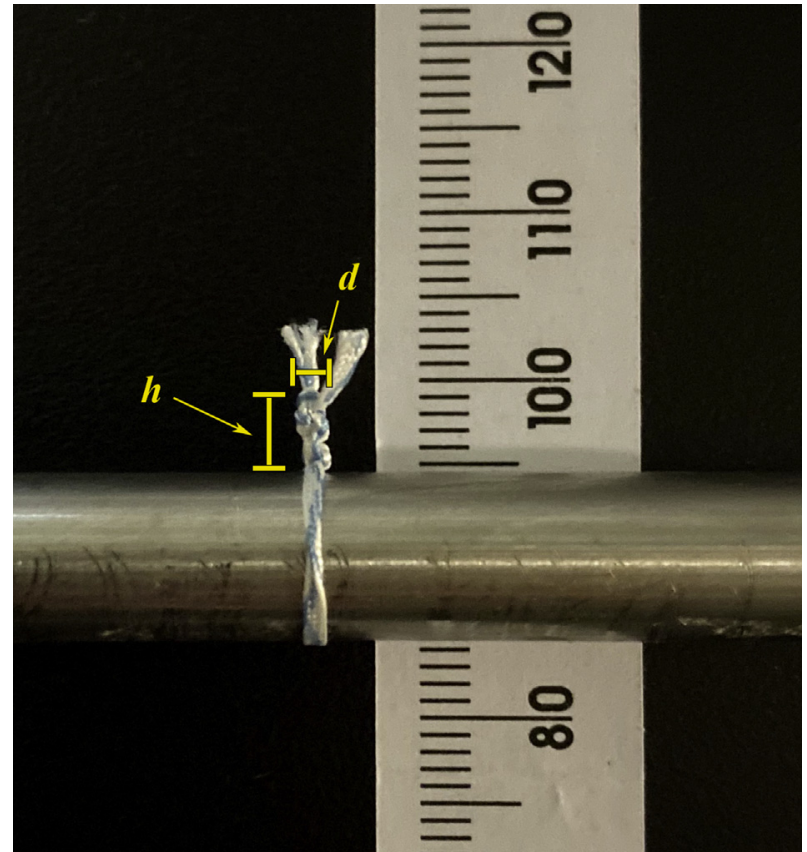

Fig 3. Representative image of the technique used to measure knot volume with a measurement diagram.

previously described by Rodes et al. ${ }^{14}$ and assumes a cylindrically shaped knot stack: $V=\frac{\pi}{4} d^{2} h$, where $V=$ knot volume, $d=$ knot diameter, and $h=$ knot height (Fig 3). Each suture-knot combination was evaluated for knot volume using 20 specimens.

\section{Statistical Analysis}

Two-way analysis of variance followed by TukeyKramer post hoc tests were used to compare the mean tensile forces reached during single load and cyclic testing, as well as the analysis of loop circumference and knot volume. Statistical comparisons were made between the larger tapes, narrow tapes, and round sutures, within groups based on the knot used. Statistical analyses were performed using Statistica software (StatSoft, Hamburg, Germany). $P<.05$ was considered statistically significant.

\section{Results}

\section{The 3-mm Single Load Testing}

In single load testing, the mode of failure varied between knot breakage and knot slippage. The mean failure load of FiberWire was lower than that of all other sutures independent of the knot tied. A combined analysis of all knots ( $\mathrm{n}=50$ for each group) showed no difference between the narrower tapes and both DynaCord and MaxBraid, but independent of the knot tied, FiberWire required significantly less force to produce $3 \mathrm{~mm}$ of loop elongation than the other sutures tested $(P<.001)$.
Individual comparisons of suture-knot combinations revealed significant differences in mean maximum load achieved by the tape sutures and round sutures across various knot types (Table 1). Hand-tied FiberTape and PermaTape achieved significantly higher loads at failure than all other hand-tied sutures $(P<.001)$. In general, the narrower tapes performed as well or better than MaxBraid and FiberWire across all knot types. Dynacord arose as the only round suture to achieve a significantly higher result over the tape sutures.

\section{2-mm Single Load Testing}

Average maximum loads between 0 and $2 \mathrm{~mm}$ of loop elongation proved similar to that seen with 3-mm displacement, albeit with expectedly lower overall maximum forces achieved (Table 2). Despite the comparable results, differences between the narrow tapes and round sutures became more apparent, with more statistically significant differences favoring the tape designs. Although PermaTape maintained its trend of statistically significant higher failure loads over all narrow tapes and round sutures, FiberTape failed at similar loads to the narrow tapes, only remaining significantly stronger than MaxBraid and FiberWire $(P<.001)$.

\section{Cyclic Testing}

All combinations demonstrated significant plastic loop deformation, or cyclic elongation, over the 1000 cycles of loading $(P<.001)$. Mean cyclic elongation ranged from 0.57 to $1.27 \mathrm{~mm}$. No suture-knot combination failed during cyclic testing. Comparisons of cyclic elongation also produced significant results, mostly favoring the tape sutures in the hand-tied group (Table 3). PermaTape and the narrow tapes performed similarly; however, FiberTape demonstrated findings more akin with the no. 2 sutures.

Load-to-3 $\mathrm{mm}$ after cyclic testing followed a similar pattern as the single load testing groups (Figure 4). The tape sutures performed as well or better than the round sutures with the exception of DynaCord (300.43 \pm $39.11 \mathrm{~N})$ failing at a significantly higher load than SutureTape $(237.72 \pm 30.05 \mathrm{~N})$ using the SMC knot $(P<.001)$.

\section{Loop Security}

After pre-tensioning to $10 \mathrm{~N}$, mean initial loop circumferences were found to range between 30.17 and $32.53 \mathrm{~mm}$, indicating a mean increase in loop size of 0.17 to $2.53 \mathrm{~mm}$ during knot tying (Table 4). Generally, the arthroscopically tied knots demonstrated better loop security than hand-tied knots. Overall, DynaCord had the lowest changes in loop circumference prior to tensile testing. Conversely, hand-tied FiberTape had a significantly larger loop circumference than each of the narrow tapes and round sutures $(P<.001)$. PermaTape 
Table 1. The 3-mm Single Load Testing (N)

\begin{tabular}{|c|c|c|c|c|c|}
\hline Suture/Knot & Hand-Tied & SMC Knot & Tennessee Slider & Duncan Loop & Surgeon's Knot \\
\hline BroadBand & $322.09 \pm 41.36^{*}, \dagger$ & $266.73 \pm 28.02^{\dagger}$ & $217.03 \pm 49.04$ & $221.73 \pm 88.40$ & $317.94 \pm 52.78$ \\
\hline SutureTape & $294.67 \pm 39.09^{\dagger}$ & $225.32 \pm 40.60$ & $204.91 \pm 50.16$ & $285.63 \pm 59.02^{\dagger}$ & $293.52 \pm 55.33$ \\
\hline MaxBraid & $263.27 \pm 37.99$ & $248.82 \pm 31.04$ & $200.18 \pm 60.27$ & $292.42 \pm 52.70$ & $271.79 \pm 73.93$ \\
\hline FiberWire & $200.30 \pm 33.34$ & $203.89 \pm 31.88$ & $182.70 \pm 49.97$ & $158.66 \pm 35.25$ & $227.48 \pm 78.51$ \\
\hline DynaCord & $331.28 \pm 22.45$ & $271.52 \pm 42.96^{\S}$ & $298.86 \pm 24.26^{\S . \|}$ & $191.37 \pm 42.57$ & $283.03 \pm 67.78$ \\
\hline
\end{tabular}

FiberTape $\quad 430.48 \pm 89.00^{9}$

PermaTape $\quad 545.16 \pm 95.09^{\natural}$

Data are presented as mean \pm standard deviation; $\mathrm{n}=10$ for all groups. Demonstrates mean maximum force (N) measured from 0 to 3 mm of displacement (or suture-knot breakage) for each suture-knot combination. $P$ values represented are within knot comparisons between tape sutures and round sutures only.

SMC, Samsung Medical Center.

*Significantly higher than MaxBraid $(P<.05)$.

${ }^{\dagger}$ Significantly higher than FiberWire $(P<.05)$.

${ }^{\ddagger}$ Significantly higher than all narrow tapes and No. 2 sutures $(P<.05)$.

${ }^{\S}$ Significantly higher than SutureTape $(P<.05)$.

"Significantly higher than BroadBand $(P<.05)$.

${ }^{\top}$ Significantly higher than DynaCord $(P<.05)$.

Table 2. The 2-mm Single Load Testing (N)

\begin{tabular}{|c|c|c|c|c|c|}
\hline Suture/Knot & Hand-Tied & SMC Knot & Tennessee Slider & Duncan Loop & Surgeon's Knot \\
\hline$\overline{\text { BroadBand }}$ & $229.48 \pm 22.90^{*}$ & $189.06 \pm 44.08$ & $155.81 \pm 56.92$ & $194.43 \pm 62.10^{t \neq}$ & $262.07 \pm 60.31$ \\
\hline SutureTape & $234.42 \pm 35.71^{*}$ & $190.19 \pm 39.27$ & $149.18 \pm 46.27$ & $228.12 \pm 41.11^{\dagger, \ddagger}$ & $234.06 \pm 89.60$ \\
\hline MaxBraid & $191.64 \pm 23.97$ & $196.60 \pm 37.57$ & $133.33 \pm 45.25$ & $213.44 \pm 41.46$ & $182.16 \pm 55.47$ \\
\hline DynaCord & $239.78 \pm 27.97$ & $205.37 \pm 24.92$ & $224.35 \pm 41.07^{\S, \|}$ & $130.21 \pm 24.82$ & $194.01 \pm 49.57$ \\
\hline FiberTape & $258.71 \pm 46.81^{*}$ & & & & \\
\hline PermaTape & $380.21 \pm 67.56^{\pi}$ & & & & \\
\hline
\end{tabular}

Data are presented as mean \pm standard deviation; $\mathrm{n}=10$ for all groups. Demonstrates mean maximum force (N) measured from 0 to 2 mm of displacement (or suture-knot breakage) for each suture-knot combination. $P$ values represented are within knot comparisons between tape sutures and round sutures only.

SMC, Samsung Medical Center.

*Significantly higher than MaxBraid $(P<.05)$.

${ }^{\dagger}$ Significantly higher than FiberWire $(P<.05)$.

${ }^{\ddagger}$ Significantly higher than DynaCord $(P<.05)$.

${ }^{\S}$ Significantly higher than SutureTape $(P<.05)$.

"Significantly higher than BroadBand $(P<.05)$.

${ }^{\top}$ Significantly higher than all narrow tapes and No. 2 sutures $(P<.05)$.

Table 3. Cyclic Elongation (mm)

\begin{tabular}{|c|c|c|c|c|c|}
\hline Suture/Knot & Hand-Tied & SMC Knot & Tennessee Slider & Duncan Loop & Surgeon's Knot \\
\hline BroadBand & $0.64 \pm 0.07^{*,+\mp}$ & $0.82 \pm 0.40$ & $1.63 \pm 0.52$ & $0.68 \pm 0.30^{\ddagger}$ & $0.97 \pm 0.66$ \\
\hline SutureTape & $0.69 \pm 0.13^{*,+\ddagger}$ & $0.84 \pm 0.31$ & $0.87 \pm 0.39$ & $0.57 \pm 0.09^{\ddagger}$ & $1.18 \pm 0.81$ \\
\hline MaxBraid & $0.95 \pm 0.10$ & $1.03 \pm 0.26$ & $1.29 \pm 0.55$ & $0.80 \pm 0.10$ & $1.35 \pm 0.47$ \\
\hline DynaCord & $0.98 \pm 0.17$ & $0.90 \pm 0.10$ & $0.97 \pm 0.22^{\S}$ & $1.22 \pm 0.35$ & $0.89 \pm 0.16$ \\
\hline FiberTape & $0.88 \pm 0.15$ & & & & \\
\hline PermaTape & $0.59 \pm 0.08^{\dagger \ddagger}$ & & & & \\
\hline
\end{tabular}

Data are presented as mean \pm standard deviation; $\mathrm{n}=10$ for all groups. Represents the mean amount of plastic loop elongation (mm) that occurred from the initial $10 \mathrm{~N}$ pre-tensioning position to the end of cyclic testing (1000 cycles). $P$ values represented are within knot comparisons between tape sutures and round sutures only.

SMC, Samsung Medical Center.

*Significantly less than FiberTape $(P<.05)$.

${ }^{\dagger}$ Significantly less than MaxBraid $(P<.05)$.

${ }^{\ddagger}$ Significantly less than DynaCord $(P<.05)$.

${ }^{\S}$ Significantly less than BroadBand $(P<.05)$. 


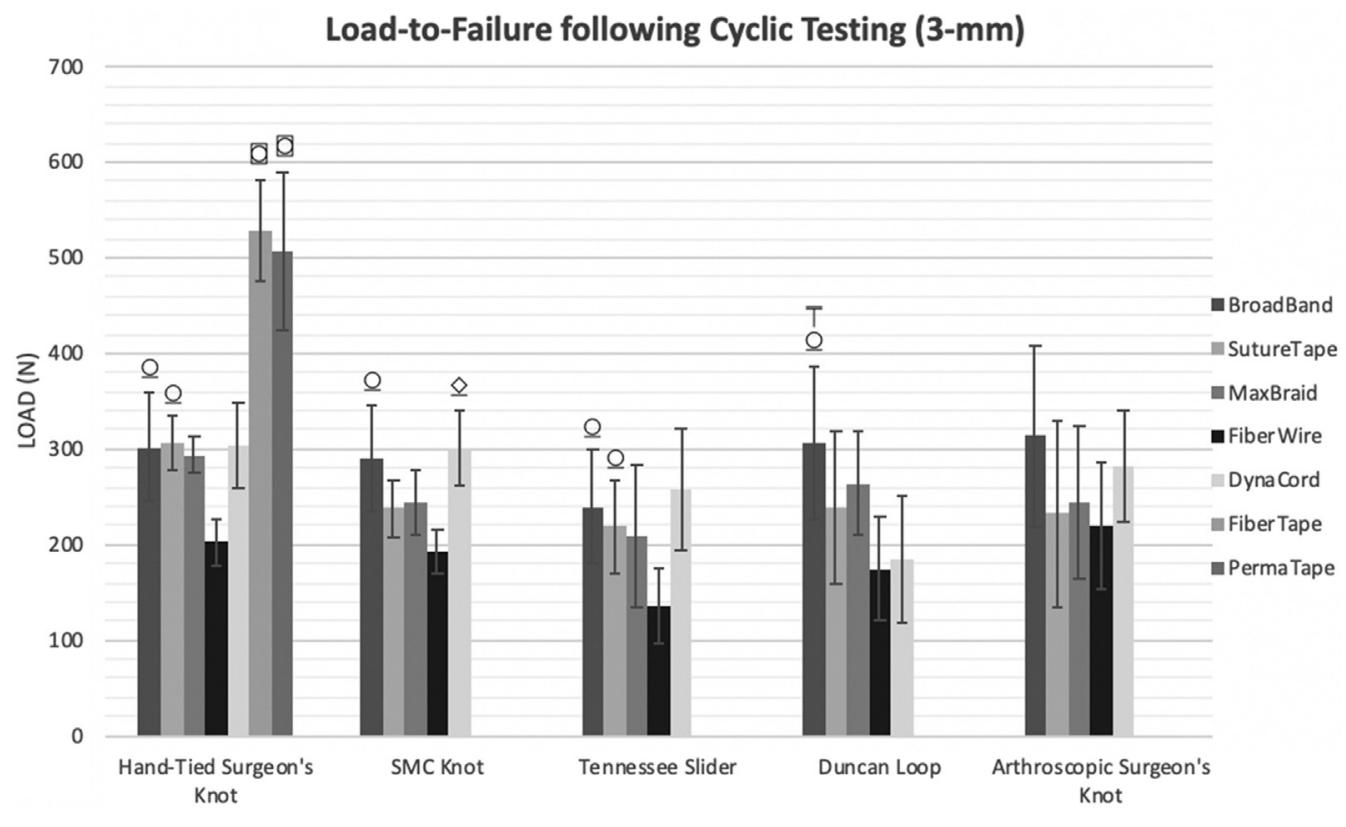

Fig 4. Load-to-failure following cyclic testing with failure defined as loop elongation of $3 \mathrm{~mm}$; vertically oriented error bars represent standard deviation; $\mathrm{n}=10$ for all groups. Mean maximum force $(\mathrm{N})$ was measured from 0 to 3 $\mathrm{mm}$ of elongation after completion of cyclic load testing. Open circles indicate values significantly higher than FiberWire $(P<$ .05). Open circles inside squares indicate values significantly higher than all narrow tapes and no. 2 sutures $(P<.05)$. Open diamonds indicate values significantly higher than SutureTape $(P<.05)$. Error bars indicate values significantly higher than DynaCord $(P<.05)$. $P$ values represented are within knot comparisons between tape sutures and round sutures only. fared slightly better, with hand-tied BroadBand, FiberWire, and DynaCord all revealing a significantly smaller circumference $(P<.001)$. The narrow tapes were generally comparable to MaxBraid and FiberWire.

\section{Knot Volume}

Comparisons of knot volume favored the narrow tape designs (Table 5). SutureTape revealed significantly smaller knot volumes with the hand-tied knot, SMC knot, Tennessee slider, and Duncan loop, than FiberWire and DynaCord $(P<.001)$. Similar results arose with BroadBand, demonstrating significantly less bulk using the hand-tied knot, SMC knot, and Duncan loop than FiberWire and DynaCord $(P<.001)$. PermaTape and FiberTape had significantly larger knot size than all of the other sutures $(P<.001)$.

\section{Discussion}

The findings observed in this study demonstrate the general reliability and strength of BroadBand and SutureTape, across multiple suture-knot combinations,

Table 4. Loop Circumference (mm)

\begin{tabular}{|c|c|c|c|c|c|}
\hline Suture/Knot & Hand-Tied & SMC Knot & Tennessee Slider & Duncan Loop & Surgeon's Knot \\
\hline BroadBand & $31.08 \pm 0.22^{*,+}$ & $30.65 \pm 0.16^{*}$ & $31.04 \pm 0.44$ & $30.47 \pm 0.40$ & $30.77 \pm 0.52$ \\
\hline SutureTape & $31.32 \pm 0.19^{\dagger}$ & $30.69 \pm 0.25$ & $30.92 \pm 0.60$ & $30.43 \pm 0.18$ & $30.76 \pm 0.28$ \\
\hline MaxBraid & $31.43 \pm 0.20^{\dagger}$ & $30.84 \pm 0.19$ & $30.94 \pm 0.25$ & $30.42 \pm 0.11$ & $30.93 \pm 0.37$ \\
\hline FiberWire & $31.25 \pm 0.21^{\dagger \neq}$ & $30.75 \pm 0.22$ & $30.95 \pm 0.43$ & $30.52 \pm 0.61$ & $30.94 \pm 0.42$ \\
\hline DynaCord & $30.19 \pm 0.19^{\S}$ & $30.26 \pm 0.14^{\|}$ & $30.20 \pm 0.19^{\|}$ & $30.17 \pm 0.24$ & $30.26 \pm 0.20^{\|}$ \\
\hline FiberTape & $32.53 \pm 0.65$ & & & & \\
\hline PermaTape & $31.61 \pm 0.26$ & & & & \\
\hline
\end{tabular}

Data are presented as mean \pm standard deviation; $n=20$ for all groups. Represents the mean circumference (mm) of the knotted suture loop after pre-tensioning to $10 \mathrm{~N}$, with the ideal circumference being $30 \mathrm{~mm}$. $P$ values represented are within knot comparisons between tape sutures and round sutures only.

SMC, Samsung Medical Center.

*Significantly less than MaxBraid $(P<.05)$.

${ }^{\dagger}$ Significantly less than FiberTape $(P<.05)$.

${ }^{\ddagger}$ Significantly less than PermaTape $(P<.05)$.

${ }^{\S}$ Significantly less than all tape sutures $(P<.05)$.

${ }^{\|}$Significantly less than BroadBand and SutureTape $(P<.05)$. 
Table 5. Knot Volume $\left(\mathrm{mm}^{3}\right)$

\begin{tabular}{|c|c|c|c|c|c|}
\hline Suture/Knot & Hand-Tied & SMC Knot & Tennessee Slider & Duncan Loop & Surgeon's Knot \\
\hline$\overline{\text { BroadBand }}$ & $11.46 \pm 1.77^{*,+,}$ & $10.05 \pm 0.76^{*,+}$ & $10.10 \pm 1.00^{\dagger}$ & $14.70 \pm 1.23^{*, \uparrow}$ & $14.23 \pm 2.51$ \\
\hline SutureTape & $10.50 \pm 1.31^{*}$, & $9.65 \pm 1.39^{*},+. \S$ & $9.85 \pm 1.71^{* \dagger}$ & $14.15 \pm 1.11^{*, \dagger}$ & $13.82 \pm 2.16$ \\
\hline MaxBraid & $12.05 \pm 1.88^{\ddagger}$ & $11.10 \pm 0.77$ & $10.79 \pm 2.25$ & $14.27 \pm 1.32$ & $14.14 \pm 2.16$ \\
\hline DynaCord & $16.82 \pm 1.67^{\ddagger}$ & $13.09 \pm 1.27$ & $14.52 \pm 2.05$ & $19.65 \pm 1.62$ & $15.54 \pm 2.62$ \\
\hline FiberTape & $70.92 \pm 5.99$ & & & & \\
\hline PermaTape & $24.32 \pm 3.40$ & & & & \\
\hline
\end{tabular}

Data are presented as mean \pm standard deviation; $\mathrm{n}=20$ for all groups. Demonstrates the mean volume $\left(\mathrm{mm}^{3}\right)$ of each suture-knot combination. $P$ values represented are within knot comparisons between tape sutures and round sutures only.

SMC, Samsung Medical Center.

*Significantly less than FiberWire $(P<.05)$.

${ }^{\dagger}$ Significantly less than DynaCord $(P<.05)$.

${ }^{\ddagger}$ Significantly less than FiberTape and PermaTape $(P<.05)$.

${ }^{\S}$ Significantly less than MaxBraid $(P<.05)$.

in several tests assessing important mechanical properties of the knotted suture loop. This level of performance and consistency was not seen among the large tapes or no. 2 sutures. Across all knot types, BroadBand and SutureTape displayed overall superior results incorporating loop integrity, knot security, and knot volume, and comparable loop security when tested against the large tape and round sutures.

Loop security represents the capability to maintain a fixed suture loop as a knot is being tied. ${ }^{6,16,17}$ The ability of the knot to resist slippage or breakage against an applied load defines knot security. ${ }^{11,16,19,21,22,24,25}$ Loop integrity combines the characteristics of knot security, or knot slippage, with the innate elastic properties of the suture material and refers to the ability of the suture-knot construct to resist forces that would otherwise result in material failure and disruption of the loop. Optimal loop integrity and security are both necessary to achieve a suture-knot construct that will provide an adequate local environment conducive with healing of the tissue repair. ${ }^{1,20-24,34}$

Applying these principals to the clinical setting proves more difficult than simple extrapolation of results. Clinical failure can occur through different mechanisms, largely grouped into 2 types. Suture (material) failure has been suggested to more likely occur due to a single maximal load, while cyclic loading will more likely lead to biologic (tissue) failure. ${ }^{9,13,16}$ Many efforts have been used to reduce clinical failures, including advancements in surgical techniques and implant innovation, as well as changes in postoperative rehabilitation protocols. ${ }^{1-5,11,13-15,19}$ This study primarily focused on properties in relation with material failure.

The exact strength of a repair construct necessary for successful tissue healing is not known and likely differs among anatomic areas and individuals. ${ }^{3,13,16,20,21,26}$ In an attempt to address this issue, Burkhart et al. ${ }^{16}$ used experimental and mathematical models to estimate the contractile force of the rotator cuff and defined $60 \mathrm{~N}$ as the amount of force that the average suture in a rotator cuff repair must be able to withstand. The suture-knot constructs tested in this study sustained forces well beyond $60 \mathrm{~N}$ before reaching failure thresholds. In the clinical scenario, however, it is best to strive for the strongest possible construct, because physiological conditions may increase or decrease the loads observed by a given suture. ${ }^{13}$

Additionally, the amount of construct elongation or displacement that a repair can tolerate while maintaining the ability to heal has not been defined in the literature. Many widely cited articles arbitrarily define a cutoff of $3 \mathrm{~mm}$ of elongation to represent clinical failure. $^{6,9,10,13,14,16,18,19,21,22,24-28,33,35,36}$ To remain consistent with other studies and because the tolerance for loss of tissue apposition with respect to healing is unclear, $3 \mathrm{~mm}$ of suture loop elongation, or suture breakage, defined clinical failure in this study. Given the possibility that healing potential requires less than 3 $\mathrm{mm}$ of gap formation, results were collected for failure defined at $2 \mathrm{~mm}$ of displacement. ${ }^{13,34,35}$

BroadBand and SutureTape largely outperformed the round sutures in single load testing, representing loop integrity. Clinically, improved loop integrity suggests greater strength of the repair construct after surgery. ${ }^{13,21,22}$ A noteworthy exception, DynaCord was the only round suture to achieve significantly greater results over any of the tape sutures. Notably the narrow tapes had more statistically significant results at $2 \mathrm{~mm}$ compared to $3 \mathrm{~mm}$ of elongation. Leishman et al. ${ }^{34}$ recorded higher forces with SutureTape over FiberWire tied with a hand-tied surgeon's knot at failure cutoffs of $2 \mathrm{~mm}(242.7 \pm 38.6 \mathrm{~N}$ and $181.2 \pm 24.4 \mathrm{~N}$, respectively) but did not reach significance at $3 \mathrm{~mm}$ $(279.3 \pm 42.4 \mathrm{~N}$ and $225.5 \pm 46.1 \mathrm{~N}$, respectively), in part because of large group variances at that elongation threshold. When comparing the same suture-knot configurations, the current study showed significantly greater forces with SutureTape at both $3-\mathrm{mm}$ and 
2-mm displacement thresholds. The statistically significant results achieved at both failure cutoffs may be attributed to increased power from a larger sample size (10 vs 6 sutures).

PermaTape and FiberTape were significantly stronger at $3 \mathrm{~mm}$ of elongation than all other sutures. Although PermaTape remained the strongest at $2 \mathrm{~mm}$, FiberTape performed more closely to the narrow tapes. FiberTape incorporates a centrally integrated polyblend core within its flat braided construction. ${ }^{3,34}$ The central core may hinder proper seating, folding, and compression of the suture within the knot, therefore having less internal friction to resist knot slippage. The other tapes in this study do not have a central core. In general, the results of loading to $3 \mathrm{~mm}$ after cyclic testing were similar to the single load testing at 3-mm displacement.

As discussed above, knot security, as assessed with single load testing, inaccurately measures knot slippage by combining it with the innate stiffness or elasticity of the suture material. To minimize the effects of elastic deformation of the suture loop with applied loads, knot security was evaluated by cyclic elongation, or the amount of plastic loop elongation that occurs through cyclic testing. ${ }^{20,28}$ The measured cyclic elongation was felt to better represent knot slippage, despite previous reports stating biologic failure, as opposed to material failure, more likely occurs via cyclic loading. 9,13,16,20 Overall, PermaTape, BroadBand, and SutureTape performed best, with the majority of statistically significant results observed in the hand-tied group. Clinically, the average cyclic elongation for all tested suture-knot combinations remained below the single load failure thresholds of 2 and $3 \mathrm{~mm}$ of elongation. This suggests that the tested configurations should adequately resist the expected forces generated in the postoperative recovery period, particularly in cases using a decelerated rehabilitation protocol. ${ }^{13,20}$

DynaCord displayed the greatest loop security, reaching statistical significance when paired with all but one knot, the Duncan loop. Increased loop security implies greater ability to approximate and maintain tissue apposition during surgical repair. ${ }^{17,21,22}$ Designed with a silicone-salt core that expands while in solution, DynaCord reduces laxity within the knotted suture loop after being tied. ${ }^{37}$ The narrow tapes had comparable loop security to the remaining No. 2 sutures, FiberWire and MaxBraid, leaving PermaTape and FiberTape with the worst results. The inferior loop security demonstrated by the large tape sutures is thought to be partially due to the initial loop circumference and the large knot base, which appeared to have difficulty seating completely, preventing the suture from conforming concentrically in a loop with a relatively small radius of curvature.

Prominent suture material, largely attributed to the knot, can also influence clinical outcomes. $^{7,8,10,11,14,15,29-31}$ Knot abrasion of the undersurface of the acromion following rotator cuff repair may affect the integrity of the repair. ${ }^{31}$ Irritation of the subcutaneous tissues and skin after Achilles' tendon repair has been described, occasionally requiring a repeat surgical procedure to address the symptoms. ${ }^{29}$ It is also widely accepted that chondral damage can occur as a result of placement of relatively large knots adjacent to an articular surface. ${ }^{14}$ Careful consideration concerning the number of securing halfhitches to provide adequate strength without excess throws increasing knot size. Minimizing knot burden while maintaining mechanical performance may improve outcomes and reduce complications related to excess suture material. The current study compared the knot volume of the large and narrow tapes and the no. 2 sutures. Expectedly, FiberTape and PermaTape exhibited the bulkiest knots, with the central core in FiberTape contributing to the largest average knot size. BroadBand and SutureTape demonstrated the smallest average knot volumes across multiple combinations. None of the round sutures were found to have significantly less knot volume than the narrow tapes.

Based on this study, BroadBand and SutureTape provided the best balance of the mechanical properties tested, along with overall smaller knot size, across a variety of configurations. However, the data suggest that the larger tapes or round sutures may be preferred over the narrow tapes in specific scenarios. The surgeon may opt to use a larger tape, with superior loop integrity, if unwavering resistance against near supraphysiologic loads are anticipated during the post-operative course. Conversely, when a significantly more intimate level of tissue apposition is of greater importance, the enhanced loop security observed in DynaCord offers potential benefit. The choice of suture repair construct ultimately depends on an understanding of the innate material properties, the mechanical characteristics of the construct, the functional interaction with biologic tissues, and the specific clinical situation. ${ }^{1-5,11}$

The advent of tape suture has improved the biomechanical properties of many surgical constructs. ${ }^{1-5}$ Other studies have demonstrated superior innate mechanical characteristics of tape designs over No. 2 suture. Taha et al. ${ }^{38}$ compared the viscoelastic properties, including stiffness, creep and deformation (plastic and elastic), of two high-strength tape designs to four round suture designs. Under static and dynamic conditions, their findings demonstrated overall superior results with tape sutures over most round sutures. $^{38}$ Although tape suture demonstrates many favorable features, novel innovations in suture technology are not limited to tape designs, as demonstrated by DynaCord. ${ }^{37}$ 


\section{Limitations}

This study is not without limitations. Some limitations may be due to the bench methods used. Attempts were made to closely simulate operative conditions; however, differences from in situ conditions existed. Laboratory conditions may generate knots of different quality than those tied under in vivo conditions. Although more controlled and precise, the mechanical testing of the suture does not directly mimic the physiological stresses after a repair. Additionally, other physiological effects, including that of the environment on the suture and the suture construct on tissue, were not evaluated. Therefore extrapolation of these findings directly to clinical results should be done with caution.

All of the arthroscopic knots were tied by a single orthopaedic surgeon, fellowship-trained in sports medicine. Although this provides consistency, knot performance could have been influenced by the surgeon's level of familiarity with each knot and the suture material and may not represent the orthopaedic community as a whole. Analysis of the innate properties of each suture material was not performed. Although this could potentially increase the accuracy in various testing (e.g., knot security and knot slippage), it was not thought to be necessary to achieve the purpose of this study. Knot size was assessed by measuring knot volume using a formula that assumes a cylindrical shape of the knot stack. This technique has not been validated in the orthopaedic literature.

\section{Conclusion}

This study exhibits the ability of narrow tape sutures to provide reliable tissue apposition and fixation in multiple suture-knot configurations, while decreasing the potential adverse effects accompanying knot prominence. Generally, BroadBand and SutureTape demonstrated an ideal balance of mechanical characteristics to achieve the optimal suture-knot construct among those tested. Dynacord displayed superior loop security and performed best among round sutures. PermaTape and FiberTape showed greater loop integrity, albeit to the detriment of loop security and knot volume.

\section{Acknowledgments}

The authors thank Arthrex, DePuy Mitek, and Zimmer Biomet for the generous donation of suture material used in this study and Luke Lindemann for his help in constructing the testing apparatus and programming the testing software.

\section{References}

1. Leger-St-Jean B, Menard J, Hinse S, Petit Y, Rouleau DM, Beauchamp M. Braided tape suture provides superior bone pull-through strength than wire suture in greater tuberosity of the humerus. J Orthop 2015;12:S14-S17 (Suppl 1).
2. Liu RW, Lam PH, Shepherd HM, Murrell GA. Tape versus suture in arthroscopic rotator cuff repair: Biomechanical analysis and assessment of failure rates at 6 months. Orthop J Sports Med 2017;5(4):2325967117701212.

3. Gnandt RJ, Smith JL, Nguyen-Ta K, McDonald L, LeClere LE. High-tensile strength tape versus high-tensile strength suture: A biomechanical study. Arthroscopy 2016;32:356-363.

4. Huntington L, Coles-Black J, Richardson M, et al. The use of suture-tape and suture-wire in arthroscopic rotator cuff repair: A comparative biomechanics study. Injury 2018;49:2047-2052.

5. Ono Y, Joly DA, Thornton GM, Lo IK. Mechanical and imaging evaluation of the effect of sutures on tendons: tape sutures are protective to suture pulling through tendon. J Shoulder Elbow Surg 2018;27:1705-1710.

6. Kuptniratsaikul S, Weerawit P, Kongrukgreatiyos K, Promsang T. Biomechanical comparison of four sliding knots and three high-strength sutures: Loop security is much different between each combination. J Orthop Res 2016;34:1804-1807.

7. Dahl KA, Patton DJ, Dai Q, Wongworawat MD. Biomechanical characteristics of 9 arthroscopic knots. Arthroscopy 2010;26:813-818.

8. Hassinger SM, Wongworawat MD, Hechanova JW. Biomechanical characteristics of 10 arthroscopic knots. Arthroscopy 2006;22:827-832.

9. Corey S, Wenger K, Johnson P, Parada S. Loop and knot security of a novel arthroscopic sliding-locking knot using high-strength sutures. J Orthop 2018;15:980-983.

10. Meyer DC, Bachmann E, Ladermann A, Lajtai G, Jentzsch $\mathrm{T}$. The best knot and suture configurations for high-strength suture material. An in vitro biomechanical study. Orthop Traumatol Surg Res 2018;104: 1277-1282.

11. Longo UG, Buchmann S, Berton A, Maffulli N, Denaro V. Arthroscopic knots and strength sutures for rotator cuff repair. Sports Med Arthrosc Rev 2011;19:251-265.

12. Abbi G, Espinoza L, Odell T, Mahar A, Pedowitz R. Evaluation of 5 knots and 2 suture materials for arthroscopic rotator cuff repair: Very strong sutures can still slip. Arthroscopy 2006;22:38-43.

13. Swan KG Jr, Baldini T, McCarty EC. Arthroscopic suture material and knot type: An updated biomechanical analysis. Am J Sports Med 2009;37:1578-1585.

14. Rodes SA, Favorito PJ, Piccirillo JM, Spivey JT. Performance comparison of a pretied suture knot with three conventional arthroscopic knots. Arthroscopy 2015;31: 2183-2190.

15. Zhao C, Hsu CC, Moriya T, et al. Beyond the square knot: A novel knotting technique for surgical use. J Bone Joint Surg Am 2013;95:1020-1027.

16. Burkhart SS, Wirth MA, Simonich M, Salem D, Lanctot D, Athanasiou KA. Knot security in simple sliding knots and its relationship to rotator cuff repair: How secure must the knot be? Arthroscopy 2000;16(2):202-207.

17. Burkhart SS, Wirth MA, Simonich M, Salem D, Lanctot D, Athanasiou KA. Loop security as a determinant of tissue fixation security. Arthroscopy 1998;14:773-776.

18. Elkousy H, Hammerman SM, Edwards, et al. The arthroscopic square knot: A biomechanical comparison 
with open and arthroscopic knots. Arthroscopy 2006;22: 736-741.

19. Elkousy HA, Sekiya JK, Stabile KJ, McMahon PJ. A biomechanical comparison of arthroscopic sliding and sliding-locking knots. Arthroscopy 2005;21:204-210.

20. Ilahi OA, Younas SA, Alexander J, Noble PC. Cyclic testing of arthroscopic knot security. Arthroscopy 2004;20: 62-68.

21. Lo IK, Burkhart SS, Chan C, Athanasiou K. Arthroscopic knots: Determining the optimal balance of loop security and knot security. Arthroscopy 2004;20:489-502.

22. Lo IK, Ochoa E Jr, Burkhart SS. A Comparison of knot security and loop security in arthroscopic knots tied with newer high-strength suture materials. Arthroscopy 2010;26(9):S120-S126.

23. Punjabi VM, Bokor DJ, Pelletier MH, Walsh WR. The effect on loop elongation and stress relaxation during longitudinal loading of fiberwire in shoulder arthroscopic knots. Arthroscopy 2011;27:750-754.

24. Shah MR, Strauss EJ, Kaplan K, Jazrawi L, Rosen J. Initial loop and knot security of arthroscopic knots using highstrength sutures. Arthroscopy 2007;23:884-888.

25. Clark RR, Dierckman B, Sampaticos N, Snyder S. Biomechanical performance of traditional arthroscopic knots versus slippage-proof knots. Arthroscopy 2013;29: 1175-1181.

26. Barber A, Herbert MA, Beavis RC. Cyclic load and failure behavior of arthroscopic knots and high strength sutures. Arthroscopy 2009;25:192-199.

27. Lieurance RK, Pflaster DS, Abbott D, Nottage WM. Failure characteristics of various arthroscopically tied knots. Clin Orthop Relat Res 2003;408:311-318.

28. Omer AI, Younas SA, Ho DM, Noble PC. Security of knots tied with Ethibond, Fiberwire, Orthocord, or Ultrabraid. Am J Sports Med 2008;36(12):2407-2414.
29. Barwick TW, Blundell CM. Reducing knot prominence during "Achillon" tendoachilles repair: Technical tip. Foot Ankle Surg 2013;19(1):e5-e6.

30. Kim SH, Ha KI. The SMC knot-A new slip knot with locking mechanism. Arthroscopy 2000;16:563-565.

31. Hanypsiak BT, DeLong JM, Simmons L, Lowe W, Burkhart S. Knot strength varies widely among expert arthroscopists. Am J Sports Med 2014;42(8):1978-1984.

32. Kim SH, Yoo JC, Wang JH, Choi KW, Bae TS, Lee CY. Arthroscopic sliding knot: How many additional half-hitches are really needed? Arthroscopy 2005:21: 405-411.

33. Pietschmann MF, Sadoghi $P$, Häuser E, et al. Influence of testing conditions on primary stability of arthroscopic knot tying for rotator cuff repair: Slippery when wet? Arthroscopy 2011;27:1628-1636.

34. Leishman DJ, Chudik SC. Suture tape with broad fullwidth core versus traditional round suture with round core: A mechanical comparison. Arthroscopy 2019;35: 2461-2466.

35. Lanzi JT Jr, Felix J, Tucker CJ, et al. Comparison of the suture anchor and transosseous techniques for patellar tendon repair: A biomechanical study. Am J Sports Med 2016;44:2076-2080.

36. Muffly TM, Cook C, Distasio J, Bonham AJ, Blandon RE. Suture end length as a function of knot integrity. J Surg Educ 2009;66:276-280.

37. Owens BD, Algeri J, Liang V, DeFroda S. Rotator cuff tendon tissue cut-through comparison between 2 hightensile strength sutures. J Shoulder Elbow Surg 2019;28: 1897-1902.

38. Taha ME, Schneider K, Clarke EC, et al. A biomechanical comparison of different suture materials used for arthroscopic shoulder procedures. Arthroscopy 2020;36: 708-713. 